\title{
Nuclear Localization Signals in Prototype Foamy Viral Integrase for Successive Infection and Replication in Dividing Cells
}

\author{
Md. Alamgir Hossain, Md. Khadem Ali, and Cha-Gyun Shin*
}

\begin{abstract}
We identified four basic amino acid residues as nuclear localization signals (NLS) in the C-terminal domain of the prototype foamy viral (PFV) integrase (IN) protein that were essential for viral replication. We constructed seven point mutants in the C-terminal domain by changing the lysine and arginine at residues $305,308,313,315,318,324$, and 329 to threonine or proline, respectively, to identify residues conferring NLS activity. Our results showed that mutation of these residues had no effect on expression assembly, release of viral particles, or in vitro recombinant IN enzymatic activity. However, mutations at residues $\mathbf{3 0 5}(\mathbf{R}$ $\rightarrow T)$, 313(R $\rightarrow T)$, 315(R $\rightarrow P)$, and 329(R $\rightarrow T$ ) lead to the production of defective viral particles with loss of infectivity, whereas non-defective mutations at residues $308(R \rightarrow$ $\mathrm{T})$, 318( $\mathrm{K} \rightarrow \mathrm{T})$, and $324(\mathrm{~K} \rightarrow \mathrm{T})$ did not show any adverse effects on subsequent production or release of viral particles. Sub-cellular fractionation and immunostaining for viral protein PFV-IN and PFV-Gag localization revealed predominant cytoplasmic localization of PFV-IN in defective mutants, whereas cytoplasmic and nuclear localization of PFV-IN was observed in wild type and non-defective mutants. However sub-cellular localization of PFV-Gag resulted in predominant nuclear localization and less presence in the cytoplasm of the wild type and non-defective mutants. But defective mutants showed only nuclear localization of Gag. Therefore, we postulate that four basic arginine residues at $305,313,315$ and 329 confer the karyoplilic properties of PFV-IN and are essential for successful viral integration and replication.
\end{abstract}

\section{INTRODUCTION}

The foamy viruses (FVs) or spumaretroviruses comprise the only genus in the subfamily Spumaretrovirinae of the family

Department of Biotechnology, Chung-Ang University, Ansung 456-756, Korea

${ }^{*}$ Correspondence: cgshin@ cau.ac.kr

Received 8 November, 2013; revised 11 December, 2013; accepted 13 December, 2013; published online 19 February, 2014

Keywords: integrase, mutation, nuclear localization signal, prototype foamy virus, retrovirus
Retroviridae (Fauquet and Fargette, 2005). Different mammalian species are natural hosts for FVs (Lecellier and Saib, 2000). Although they are non-pathogenic to their hosts, FVs can infect a wide range of tissues under cell cuture conditions and show cytopathic effects through syncytia formation and extensive vacularization (Lo et al., 2010). The FV replication cycle differs from orthoretroviruses in many aspects (Linial, 2007; Rethwilm, 2005) such as their mode of pol protein expression (Löchelt and Flugel, 1996; Pearl and Taylor 1987), requirements of cycling cells for gene expression (Patton et al., 2004), the presence of large amounts of infectious DNA, and their RNA genome (Linial, 1999; Rethwillm, 1996; Yu et al., 1999). The lack of FV pathogenicity has prompted their potential use as a safer gene therapeutic vector, and a variety of vectors for different cell types are available (Mergia and Heinkelein, 2003). The prototype foamy virus (PFV), previously known as human foamy virus was isolated from a Kenyan patient with nasopharynx carcinoma (Achong et al., 1971). High sequence homology to FVs from chimpanzee and the lack of evidence of natural human infections strongly suggests that this virus was derived by zoonotic transmission from a chimpanzee (Epstein, 2004; Herchenroder et al., 1994; Mahnke et al., 1990; Schweizer et al., 1995).

Viruses in the family Retroviridae contain RNA as their genome. They must reverse transcribe their genome to doublestranded cDNA for successful infection, replication in their hosts, and to integrate into the host cell chromosome (Brown, 1997). As integration occurs in the nucleus, a large nucleoprotein complex termed the preintegration complex (PIC) tethers the viral cDNA from the cytoplasm to the nucleus (Wei et al., 1997). Previous studies have shown that passive diffusion of $50 \mathrm{kDa}$ proteins into the nucleus occurs via the aqueous channel of the nuclear pore complex (Gorlich and Kutay, 1999; Mattaj and Englmeiier, 1998). Although nuclear localization of several retroviral INs has been described in the context of transfection assays, the identity of the NLS is not well defined and the mechanism of importation remains poorly understood (BouyacBertoia et al., 2001; Katz et al., 2002; Petit et al., 2000).

Retroviral integrases (INs) have two important roles in the viral life cycle. They tether viral cDNA from the cytoplasm to the nucleus, as it contains nuclear localization signals (NLS) (Craigie, 2001) and catalyze integration into the cellular genomic DNA (Bushman et al., 1990; Engelman et al., 1991). HIV-1 IN has a basic bipartite type NLS at residues 186-188 and 211-219 
(Gallay et al., 1997). A separate study reported an additional NLS in the central domain of the HIV-1 IN at residues 161-173 (Bouyac-Bertoia et al., 2001). The karyophilic determinant of the feline immunodeficiency virus (FIV) IN has been mapped to the highly conserved $\mathrm{N}$-terminal zinc binding $\mathrm{HHCC}$ motif (Woodward et al., 2003). A functional NLS of the avian sarcoma virus IN has been mapped to residues 206-235 (Kukolj et al., 1997). An active foamy virus IN is essential for viral replication and successful infection (Enssle et al., 1999). Studies have revealed that foamy virus Gag and IN contain NLS sequences (Mullers et al., 2011). The NLS sequence in the Gag protein is responsible for its translocation and higher accumulation in the nucleus (Schliephake and Rethwilm, 1994; Yu et al., 1996). Using IN-specific monoclonal antibodies, the IN domain of the Pol protein has an NLS, responsible for its importation into the nucleus (Imrich et al., 2000). Although it has been suggested to be part of PFV PIC, the Gag protein translocates to the cell nucleus and facilitates integration by tethering the viral DNA genome to the host cell chromatin (Tobaly-tapiero et al., 2008). A separate study showed that translocation of Gag and the viral genome to the nucleus is dependent on IN in both cycling and growth arrested cells (Lo et al., 2010).

In order to study any relationship between NLS resides of PFV-IN and viral replication, seven point mutations at the Cterminal region of PFV-IN has been made. All the tested residues are arginine and lysine, which were regarded as NLS residues since they are present at the conserved region of the C-terminal region. In this study, we utilized the full-length PFV constructs, which contain point mutation, and analyzed their effects on viral assembly, release, and infectivity. We also analyzed the necessity of these residues for efficient post-entry nuclear localization of the virion-associated IN and Gag proteins. We concluded that amino acids at the positions 305, 313, 315 , and 329 are critical for nuclear localization of PFV-IN. Mutation at these positions totally abolished viral infectivity. Therefore we propose that these NLS are essential for nuclear importation of PFV-IN through the nuclear pore complex and subsequent integration of the viral genome.

\section{MATERIALS AND METHODS}

\section{Cells and cell culture}

The BHK-21 (baby hamster kidney) cell line was obtained from the American Type Culture Collection (USA) and maintained in Dulbecco's Modified Minimal Essential Medium supplemented with $10 \%$ fetal bovine serum, $2 \mathrm{mM} \mathrm{L-glutamine,} 100 \mathrm{U}$ of penicillin $/ \mathrm{ml}$, and $100 \mu \mathrm{g}$ of streptomycin $/ \mathrm{ml}$. The FAB cell line was kindly donated by Drs Maxine L. Linial and Eun-Gyung Lee (Fred Hutchinson Cancer Research Center, USA) and maintained in $100 \mu \mathrm{g} / \mathrm{ml}$ hygromycin.

\section{Construction of mutants}

Molecular cloning was performed according to standard techniques (Rothnie et al., 1994). For in vitro expression of IN mutant proteins, PFV-IN DNA fragments containing mutation were created via overlapping PCR with the mutagenic primers described in Table 1 (Lee et al., 2005), and ligated into the Ndel/ BamHI sties of $\mathrm{pET} 15 \mathrm{~b}$. Briefly, in order to construct the mutant M 305, first PCR was performed to amplify the half fragment with the primers, PFINS and PFIN305A, and the other half fragment with the primers, PFIN305S and PFINA using proviral DNA (pHSRV) as a template (Löchelt et al., 1995). Next, PCR was carried out using PFINS and PFINA in the presence of the two half fragments generated from the first PCR. The amplified full length DNA of PFV-IN, which contained a point mutation at the 305th amino acid, was directly cloned into pET15b after restriction digestion with $\mathrm{Ndel}$ and BamHI. The other mutants were also prepared via same methods using the corresponding primers described in Table 1. The mutations were verified by DNA sequencing conducted by Macrogen, Korea.

The proviral DNA which contains the point mutation at the PFV-IN DNA was constructed by replacing the fragments of $\mathrm{Mscl} /$ Aflll region of an intermediate vector pBS-FIN-spel with the same region of the mutant-containing pET16b-PFV-IN, and then by transferring the Spel fragment of the pBS-FIN-spel into the pHSRV.

Purification and in vitro assays for PFV- IN protein The DNA constructs were transformed into E. coli (BL21 DE3) for in vitro expression of the NLS-mutated IN protein, and IN protein expression was induced with isopropyl-1-thio- $\beta$-D-galactopyranoside. The IN proteins were purified by NTA-chromatography and SP-Sepharose chromatography, as described previously (Kang et al., 2008). In vitro catalytic activities of the purified IN proteins were investigated by studying endonucleolytic and disintegration activity. Briefly, ${ }^{32} \mathrm{P}$-radio-labeled $20 \mathrm{mer}$ oligonucleotide, whose sequence mimics the PFV U5 LTR end, was annealed with a complementary oligonucleotide. Active IN protein converts the substrate (the radio-labeled 20mer/unlabeled 20mer) into the product (the radio-labeled 18mer/unlabeled 20mer). The product was visualized as the $18 \mathrm{mer}$ on a $20 \%$ polyacrylamide denaturing gel (Kang et al., 2008; Lee et al., 2005). For the disintegration assay, ${ }^{32} \mathrm{P}$-radio-labeled $15 \mathrm{mer}$ oligonucleotide was annealed with three different oligonucleotides to form a Y-shaped substrate (Lee et al., 2005). Active IN protein converts the radio-labeled 15 mer substrate into the product of the radio-labeled $30 \mathrm{mer}$. The product is visualized as the 30 mer on a $20 \%$ polyacrylamide denaturing gel (Lee et al., 2005).

Transfection and production of virus stocks

Unless otherwise stated, about $2.4 \times 10^{6}$ BHK-21 cells were grown on a $60 \mathrm{~mm}$ tissue culture plate. After $24 \mathrm{~h}$ or when the culture reached approximately $70 \%$ confluency, cells were transfected using the TrueFect (United Biosystems, USA) transfection reagent, according to the manufacturer's protocol. The culture supernatant generated by transient tranfection for the infection experiment was harvested, passed through a $0.45 \mu \mathrm{m}$ filter, and stored at $-80^{\circ} \mathrm{C}$ for future use.

Infection and titration of virus

Viruses were prepared by transfection of BHK-21 cells seeded in $60 \mathrm{~mm}$ dishes with $2.5 \mu \mathrm{g}$ of the corresponding proviral PFV plasmids. About $1 \mathrm{ml}$ of collected cell-free culture supernatant was used to infect $1.6 \times 10^{6} \mathrm{BHK}-21$ cells in $35 \mathrm{~mm}$ tissue culture plates. A FV-activated $\beta$-galactosidase (Gal) expression assay (called a blue assay) was performed, as described by $\mathrm{Yu}$ and Linial, to evaluate infectivity of the virus prepared by transfection (Yu and Linial, 1993). Briefly, the FAB cell line has a single integrated copy of a long terminal repeat (LTR)-driven by the $\beta$-Gal gene. Viral infection causes the PFV LTR promoter to be transactivated by the viral Tas protein, resulting in a blue color in the infected cells after X-gal staining. A $1.5 \times 10^{4}$ aliquot of $F A B$ cells was infected with the appropriate dilutions of virus containing cell-free culture supernatant after $24 \mathrm{~h}$ of seeding in 48-well culture plates. The total number of blue cells was then counted after an additional 2-day-incubation. 
PFV Integrase NLS for Infection

Md. Alamgir Hossain et al.

Table 1. PCR primers

\begin{tabular}{|c|c|c|}
\hline Primers & Sequences $\left(5^{\prime}-3^{\prime}\right)$ & Restriction or mutagenic sites \\
\hline PFINS & 5'-GGCAGCCATATGAAAGGATATCCC-3' & Ndel \\
\hline PFINA & 5'-GCGCGGATCCTTATTCATTTTTTTCCAAATG-3' & BamHI \\
\hline PFIN305S & 5'-CAATTGGTCCAGGAGACGGTGGCTAGGCCTGCTTC-3' & M305 $(R \rightarrow T)$ \\
\hline PFIN305A & 5'-AGCAGGCCTAGCCACCGTCTCCTGGACCAATTG-3' & M305 $(R \rightarrow T)$ \\
\hline PFIN308S & 5'-CAGGAGAGGGTGGCTACGCCTGCTTCTTTGAGAC-3' & M308 $(R \rightarrow T)$ \\
\hline PFIN308A & 5'-TCTCAAAGAAGCAGGCGT AGCCACCCTCTCCTG-3' & M305 $(R \rightarrow T)$ \\
\hline PFIN313S & 5'-AGGCCTGCTTCTTTGACACCTCGTTGGCATAAAC-3' & M313 $(R \rightarrow T)$ \\
\hline PFIN313A & 5'-TTTATGCCAACGAGGTGTCAAAGAAGCAGGCCTAG -3' & M313 $(R \rightarrow T)$ \\
\hline PFIN315S & 5'-GCTTCTTTGAGACCTCCATGGCATAAACCGTCTACTG-3' & M315 $(R \rightarrow P)$ \\
\hline PFIN315A & 5'-GTAGACGGTITATGCCATGGAGGTCTCAAAGAAGCAGG-3' & M315 $(\mathrm{R} \rightarrow \mathrm{P})$ \\
\hline PFIN318S & 5'-AGACCTCGTTGGCATACACCGTCTACTGTACTTAAG-3' & M318 $(\mathrm{K} \rightarrow \mathrm{T})$ \\
\hline PFIN318A & 5'-AAGTACAGTAGACGGTGTATGCCAACGAGGTCTC-3' & M318 $(\mathrm{K} \rightarrow \mathrm{T})$ \\
\hline PFIN324S & 5'-CTACTGTACTTACGGTGTTGAATCCAAGGACTGTTG-3' & M324 $(\mathrm{K} \rightarrow \mathrm{T})$ \\
\hline PFIN324A & 5'-CAACAGTCCTTGGATTCAACACCGTAAGTACAGTAG-3' & M324 $(\mathrm{K} \rightarrow \mathrm{T})$ \\
\hline PFIN329S & 5'-CTTAAGGTGTTGAATCCAACGACTGTTGTTATTTTG-3' & M329 $(R \rightarrow T)$ \\
\hline PFIN329A & 5'-CAAAATAACAACAGTCGTTGGATTCAACACCTTAAG-3' & M329 $(R \rightarrow T)$ \\
\hline
\end{tabular}

Mutagenic sequences are indicated in italics. Restriction sites are underlined.

Assay of virion-associated reverse transcriptase (RT) activity

Virion-associated RT assays were performed as described previously (Rinke et al., 2002). RT activity was determined by incorporation of [ $\left.{ }^{3} \mathrm{H}\right] \mathrm{TTP}$ using a poly $(\mathrm{A}):(\mathrm{dT})_{12-18}$ (Midland Certified Reagent Co., USA) as the template-primer. Viral supernatants from infected BHK21 cells were filtered, and virions were pelleted through a $20 \%$ sucrose cushion by centrifugation at $25,000 \times g$ for $1 \mathrm{~h}$. A $10 \mu \mathrm{l}$ aliquot of the virion was added to 50 $\mu \mathrm{l}$ of reaction cocktail, consisting of $45 \mathrm{mM}$ Tris $(\mathrm{pH} 8.0), 50 \mathrm{mM}$ $\mathrm{NaCl}, 2.5 \mathrm{mM} \mathrm{MgCl}_{2}, 5 \mathrm{mM}$ DTT, $10 \mu \mathrm{g}$ of poly(A):(dT) ${ }_{12-18}$ per $\mathrm{ml}, 0.1 \% \mathrm{NP}-40$, and $1 \mu \mathrm{l}$ of $[3 \mathrm{H}] \mathrm{TTP}$. The reaction mixtures were incubated at $37^{\circ} \mathrm{C}$ for $1 \mathrm{~h}$. Then, the mixtures were transferred to DE81 filters (Whatman), and the filters were washed in $2 \times$ SSC (0.3 M sodium chloride, $30 \mathrm{mM}$ sodium citrate) in $95 \%$ ethanol and dried. Radioactivity was counted in a Beckman scintillation counter (LC6000SC).

\section{Western blotting}

About $1.6 \times 10^{6} \mathrm{BHK}-21$ cells were grown in $35 \mathrm{~mm}$ tissue culture plates and transfected as described above with $1.5 \mu \mathrm{g}$ of proviral PFV constructs for Western blot detection of viral protein in transfected cell lysates. Forty-eight hours after transfection, the medium was aspirated from the wells, and cells were washed once with PBS. About $150 \mu \mathrm{l}$ of antibody buffer [20 mM Tris ( $\mathrm{pH} 7.4$ ), $50 \mathrm{mM} \mathrm{NaCl}, 0.5 \%$ deoxycholic acid, $0.5 \%$ sodium dodecyl sulfate (SDS), 1 mM EDTA ( $\mathrm{pH} 8.0$ )] was added dropwise onto the cell monolayer, kept for $5 \mathrm{~min}$ on ice, scraped, placed in a $1.5 \mathrm{ml}$ Eppendorf tube, and then passed through a 23-gauge needle for mechanical shearing. Finally, cell lysates were centrifuged at $13,000 \mathrm{rpm}$ for $10 \mathrm{~min}$ using a tabletop microcentrifuge to isolate the clear lysates. Protein amount was measured by the Bradford assay. The culture supernatant was harvested, passed through $0.45 \mu \mathrm{m}$ filter, and centrifuged at $4^{\circ} \mathrm{C}$ and $17,000 \mathrm{rpm}$ for $1 \mathrm{~h}$. Viral pellets were then resuspended in antibody buffer and stored at $-80^{\circ} \mathrm{C}$. After adding an appropriate amount of SDS sample loading buffer to cell lysates and/or viral pellets, the proteins were resolved on $12.5 \%$ SDS-polyacrylamide gel electrophoresis. The proteins were then transferred to Immobilon- $P$ membranes (Millipore, USA). The membranes were blocked with $5 \%$ non-fat milk solution, and hybridized with antibodies in a PBS solution containing $0.5 \%$ Tween 20 , followed by a final rinse in PBS- $1.0 \%$ Tween 20. The proteins were immunoblotted with 1:1,000 and 1:5,000 dilutions of rabbit polyclonal antibody against PFV-IN and PFV-Gag and a secondary donkey anti-rabbit horseradish peroxidase-conjugated antibody diluted 1:10,000. Detection was carried out with a horseradish peroxidase detection kit (Westsave Gold, Ab Frontier, Korea), according to the manufacturer's instructions. Signals were detected using an X-ray film developer system.

\section{Sub-cellular fractionation}

Cellular contents were fractionated into cytoplasmic and nuclear fractions following a method described previously (Holden and Horton, 2009) with the following modifications. About $1.6 \times$ $10^{6}$ BHK-21 cells were seeded onto a $60 \mathrm{~mm}$ tissue culture plate. After $24 \mathrm{~h}$, the cells were infected with $1 \mathrm{ml}$ of cell-free culture supernatant as virus stock for $6 \mathrm{~h}$. After the incubation, the cells were rinsed with $1 \mathrm{ml}$ PBS and then $150 \mu$ ldigitonin buffer [150 mM NaCl, 50 mM HEPES (pH 7.4), $50 \mu \mathrm{g} / \mathrm{ml}$ digitonin (Sigma, USA)] was added dropwise onto the cell monolayer, kept for $5 \mathrm{~min}$ on ice, scraped, placed in a $1.5 \mathrm{ml}$ Eppendorf tube, and then mixed by gentle pipetting. After a 10 min incubation at $4^{\circ} \mathrm{C}$, the cell suspension was centrifuged at $5,000 \mathrm{rpm}$ for $5 \mathrm{~min}$, and the supernatant was collected as the cytosolic fraction. Then, the pellets were dissolved into $150 \mu \mathrm{l} \mathrm{NP40}$ buffer [150 mM NaCl, $50 \mathrm{mM}$ HEPES (pH 7.4), 1\% NP40], incubated for $30 \mathrm{~min}$ on ice, and centrifuged at $9000 \mathrm{rpm}$ for 5 min. The supernatant was discarded and the pellet was dissolved in $150 \mu \mathrm{l}$ RIPA buffer [ $150 \mathrm{mM} \mathrm{NaCl}, 50 \mathrm{mM} \mathrm{HEPES} \mathrm{pH}$ (7.4), $0.5 \%$ sodium deoxycholate, and $0.1 \%$ SDS] and incubated for $1 \mathrm{~h}$ at $4^{\circ} \mathrm{C}$. Finally, the suspension was centrifuged to remove the insoluble protein as the pellet, and the supernatant 
A

(1) Endonucleolytic activity

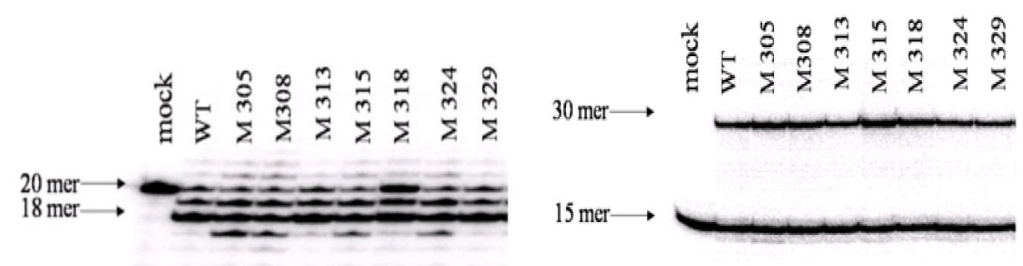

B


Fig. 1. Analyses of in vitro activities, processing, and assembly of the viral protein into the virion. (A) In vitro enzymatic activities of purified prototype foamy viral integrase (PFV-IN). Plasmids harboring both wild type and point mutated PFV-IN were used to transform $E$. coli BL-21. The cells were then grown, induced and recombinant PFV-IN was purified. The catalytic activities of the purified IN proteins were assessed by endonucleolytic and disintegration assays with respective substrates, completed reactions were resolved on a denaturing gel, and band separation were observed by autoradiography. (B) Detection of intracellular and extracellular viral proteins by Western blot analyses. Whole cell lysates and viral pellets were prepared from BHK-21 cells 48 and $72 \mathrm{~h}$ after transfection with wild type and mutant provirus expression vectors. The same amount of protein were resolved on $12.5 \%$ sodium dodecyl sulfatepolyacrylamide gels, transferred to a membrane, and then probed with anti-IN and anti-Gag antibodies. Detection of cellular protein $\beta$-actin was used as the loading control.

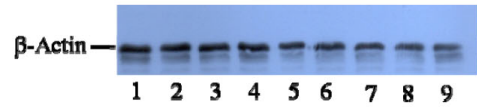

was kept as the nuclear fraction.

\section{Immunohistochemistry}

A $10^{4}$ aliquot of BHK-21 cells was grown on glass coverslips coated with $50 \mu \mathrm{g} / \mathrm{ml}$ poly(D) lysine (Sigma-Aldrich) in $35 \mathrm{~mm}$ tissue culture plates for immunostaining detection of viral protein localization in cells. Twenty-four hours after seeding, the cells were rinsed with PBS and infected with $1 \mathrm{ml}$ of cell-free culture supernatant for $6 \mathrm{~h}$. After the infected cells were incubated for the desired length of time, they were rinsed once with PBS and then fixed in 3\% paraformaldehyde for 10 minutes at room temperature. After rinsing with PBS-GSA (10 mM glycine and $0.2 \%$ sodium azide), the cells were permeabilized with $0.5 \%$ Triton-X-100 for exactly $3 \mathrm{~min}$. After blocking (3\% bovine serum albumin in PBS-GSA), coverslips were successively incubated with rabbit polyclonal anti-PFV-IN (1:40) or rabbit polyclonal anti-PFV-Gag (1:150) for $1 \mathrm{~h}$. Cells were then washed and incubated for 30 min with 1:80 (PFV-IN) or 1: 200 (PFVGag) dilutions of FITC-conjugated donkey anti-rabbit IgG (sc2089, Santa Cruz Biotechnology Inc., USA) secondary antibody. Finally, the coverslips were mounted onto a microslide using a drop of UltraCruz mounting medium (sc-24941, Santa Cruz Biotechnology) with DAPI. Fluorescence microscopy observations were performed with a fluorescence microscope (Nikon, Eclipse, TE 2000-U, Japan) at a magnification of 400x.

\section{RESULTS}

Inactivation of IN NLS does not affect IN enzymatic function, viral expression, or viral release

It has been suggested that IN integrity is not only important for mediating integration but also important for virus assembly, morphogenesis, and efficient reverse transcription (Engelaman,
1999). Thus, it was necessary to determine whether the mutations in the conserved amino acids affected IN enzymatic function through protein misfolding. To assess the structural integrity of the IN C-terminal domain, we measured the IN catalyzed in vitro endonucleolytic activity and disintegration activity using purified recombinant proteins expressed in $E$. coli. Results of both assays (Fig. 1A) indicated that the NLS mutated IN proteins were as active as the wild type proteins, indicating that mutations at those positions did not alter enzyme activity.

Next, all constructs including the wild type and mutants were transfected into BHK-21 cells to evaluate expression, assembly, and release of virus. Cells were harvested at $48 \mathrm{~h}$ post-transfection, and the presence of viral IN and Gag in transfected cell lysates was determined by Western blotting analysis. Cell lysates from mutant constructs showed similar IN and Gag processing to that of the wild type (Fig. 1B), indicating that mutations in these residues did not affect viral construct expression because production of viral particles from transfected constructs does not depend on the NLS of PFV-IN. Cellular expression machinery leads to the expression of viral construct regardless of the mutations. We collected the supernatants after $72 \mathrm{~h}$ of transfection to analyze the mutational effects on subsequent assembly and morphogenesis of viral particles. A similar IN and Gag detection level in the wild type and mutant viral lysates (Fig. 1B) indicated that all constructs successfully expressed, assembled, and released the viral particles into the culture supernatant irrespective of mutation. Therefore, these results indicate that IN mutations at these NLS positions did not affect IN enzymatic function, viral expression, or viral release. 
IN NLS is required for successful infection of cycling cells The significant role of the IN NLS for PFV replication was tested by challenging BHK-21 cells with wild type or mutant viruses. We collected the cell-free virions from the culture supernatants of the BHK-21 cells transfected with wild type and mutant constructs to assess infectivity of the released viral particles. The presence of viral particles in the culture supernatant was confirmed by viral IN and Gag Western blotting (Fig. 1B).

We challenged the $F A B$ indicator cells with the appropriate dilution of culture supernatant and measured the viral titer as the number of blue cells per milliliter to measure infectivity in a more quantitative fashion. The $\beta$-gal reporter gene is under the transcriptional control of a PFV LTR in the FAB indicator cell line, such that expression is induced by PFV infection as a consequence of viral DNA synthesis. Therefore, the lack of blue cells due to the NLS mutation reflected a lack of viral DNA integration as the virions possesses defective NLS that results failure of viral cDNA transfer from cytoplasm to nucleus. As shown in the Table 2, M 308, M 318, and M 324 more or less produced similar numbers of blue cells compared to those of the wild type. Therefore, mutations at those positions did not significantly affect viral infectivity. However very few or an undetectable number of blue cells were found for M 305, M 313, M 315 and $M 329$, indicating that mutations at those positions seriously affected viral infectivity due to the absence of functional NLS. Finally, we categorized our seven point mutants into two groups based on their ability to produce blue cells. We termed defective mutants as those point mutants (M 305, M 313, M 315, and M 329) in which mutation seriously interfered with their infectivity, and non-defective mutants as those mutant viruses (M 308, M 318, M 324) in which mutation had very little or no effect on infectivity.
Table 2. Infectivity of viruses present in supernatants of cells transfected with NLS mutant constructs

\begin{tabular}{cc}
\hline DNA constructs & Blue cells $/ \mathrm{ml}^{\mathrm{a}}$ \\
\hline WT & $(2.3 \pm 0.7) \times 10^{5}$ \\
M 305 & $(1.8 \pm 0.4) \times 10^{3}$ \\
M 308 & $(2.0 \pm 0.8) \times 10^{4}$ \\
M 313 & $160 \pm 30$ \\
M 315 & $<10$ \\
M 318 & $(4.6 \pm 0.8) \times 10^{5}$ \\
M 324 & $(3.4 \pm 0.5) \times 10^{5}$ \\
M 329 & $240 \pm 25$ \\
\hline
\end{tabular}

anfectivity of the viruses present in supernatants of the cell culture was measured by FAB assay as described in "Materials and Methods". Data are shown as the average \pm standard deviation from three independent assays.

We challenged BHK-21 cells with the culture supernatant that contained viruses to determine the effect of NLS mutation on viral replication by incubating them for 2,4 , and 6 days. Then, we harvested the supernatants to collect cell-free virions. The amount of virions was analyzed by virion-associated RT assay and Gag Western blotting. The non-defective mutants including the wild type continuously produced virions, as shown by the higher values in the RT assay and Gag presence on Western blot, while the defective mutants failed to produce cell-free virions, as they showed background levels in the RT assay and Gag was not detected by Western blot (Figs. 2A and 2B). It was very interesting how the defective mutant could not produce cell-free virions in the infection experiments even though they were produced in transfected cells.


Fig. 2. Effect of mutations on viral replication. (A) Virionassociated reverse transcriptase (RT) activity of wild type and mutant viral particles. BHK-21 cells were infected with $1 \mathrm{ml}$ of released virus from the transfection experiment. Extracellular virions were harvested at 2, 4, and $6 \mathrm{~d}$ post-infection, concentrated by centrifugation, and RT activity was assessed by measuring incorporation of $\left[{ }^{3} \mathrm{H}\right] \mathrm{TTP}$ on a poly $(\mathrm{A})$ :(dT) ${ }_{12-18}$ templateprimer. Data are an average of three independent experiments. (B) Detection of Gag in the virion pellet. The same number of virion pellets was resolved on $12.5 \%$ sodium dodecyl sulfatepolyacrylamide gels, transferred to a membrane, and then probed with anti-Gag antibody. 
Differential localization of PFV-IN and PFV-Gag

We further examined the localization of IN by sub-cellular fractionation of newly infected cells to determine the cause for the lack of blue cells in the defective mutant viruses (Fig. 3). BHK21 cells were challenged with the same amount of culture supernatant from the same collection and incubated for 24, 48, and $72 \mathrm{~h}$. After each incubation period, the cell lysates were fractionated into cytoplasmic and nuclear fractions. The localization of wild type or mutant IN proteins in these fractions was measured by Western blotting. The reason for considering IN as the preferred protein to monitor lies in its obligate presence and tight association with PICs (Miller et al., 1997).

Wild type IN was localized in the cytoplasmic fraction $24 \mathrm{~h}$ after infection (Fig. 3A). At 48 and $60 \mathrm{~h}$ after infection, the presence of IN was detected in both the cytoplasmic and nuclear fractions and increased time-dependently. All non-defective mutants showed similar IN distribution as that shown by the wild type (Fig. 3A). In contrast, the IN proteins in defective mutant viruses accumulated predominantly in the cytoplasmic fraction, and were marginally detected in the nuclear fractions (Fig. 3A). These results suggest that the loss of IN localization in the nucleus in the defective mutant viruses disrupted integration of viral DNA into the host cell chromosome, and no blue cells were produced.

FV Gag has an NLS sequence that is responsible for its translocation into the nucleus, although the cause for this accumulation is still unknown (Schliephake and Rethwilm, 1995; Yu et al., 1996). We detected sub-cellular localization of the Gag protein by Western blotting to demonstrate Gag localization in newly infected cells for both the defective and nondefective NLS mutant viruses. We utilized the same cellular fractions with the same amount of protein as used for PFV-IN detection. As shown in Fig. 3A, Gag protein expression increased time dependently during the wild type and non-defective mutant viral infection. Beginning $24 \mathrm{~h}$ post infection, Gag translocated to the nucleus, and higher accumulation of Gag proteins were observed in the nucleus at $48 \mathrm{~h}$ post infection. The possible cause for this gradually increased Gag was the integration of the viral genome into the host cell genome and expression of viral proteins. However, differential Gag translocation patterns were observed for those infected with defective NLS mutant viruses. At $24 \mathrm{~h}$ post infection Gag translocated into the nucleus of infected cells, but no accumulation was observed in the cytoplasmic fraction. We also observed a uniform amount of Gag in the nucleus, indicating that no further expression and translocation occurred for these mutant viruses.

We examined sub-cellular localization of viral IN and the Gag protein to more directly address the karyophylic properties of PFV-IN NLS mutants. BHK-21 cells were infected with the virus stocks and subjected to indirect immunofluorescent staining. PFV-IN was exclusively localized in the cytoplasm in the wild type viruses at $24 \mathrm{~h}$ post infection while fluorescence signals originated from both the cytoplasm and nucleus at $48 \mathrm{~h}$ and 72 $\mathrm{h}$ post infection (Fig. 3B), indicating translocation of IN from the cytoplasm to the nucleus and synthesis of new viral INs. Then, we examined sub-cellular localization of IN for M 315 and M 329 as representative defective mutants. As expected from the sub-cellular fractionation results, the IN protein fluorescence uniformly originated from the cytoplasm at 24,48 , and $72 \mathrm{~h}$ post infection. Thus, we confirmed that mutations at those positions caused loss of the karyophilic property. Finally, we examined the localization of two non-defective mutants M 318 and $\mathrm{M} 324$. We found that these IN mutants were almost predominantly present in the cytoplasm at $24 \mathrm{~h}$ post infection and
A



B
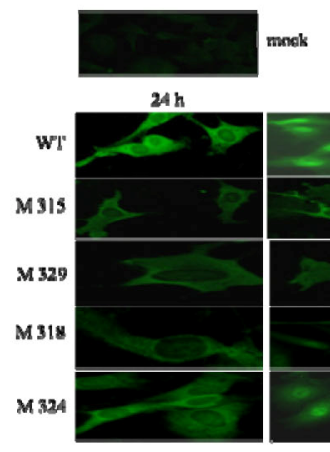

$48 \mathrm{~h}$



72 b

C

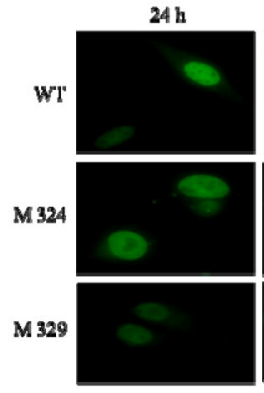

$48 \mathrm{~h}$

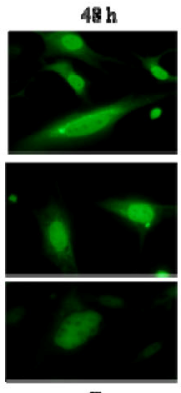

Gages

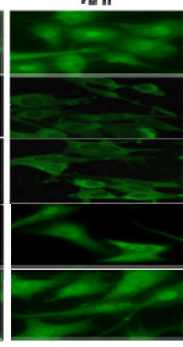

$7 \mathrm{~h}$



Fig. 3. Sub-cellular localization of prototype foamy viral integrase (PFV-IN) and PFV-Gag. (A) Western blotting analysis for subcellular localization. BHK-21 cells were infected with $1 \mathrm{ml}$ of culture supernatant containing wild type and mutant viruses and harvested after the indicated incubation times. Cells were then lysed and fractionated into cytosolic and nuclear portions as described in materials and methods. An equal amount of the fractions was resolved by $12.5 \%$ SDS-PAGE. Western blot analyses were performed with anti-IN, anti-Gag and anti-caspase-3 (cytosolic control) antibodies. (B) Immunostaining analysis for PFV-IN. (C) Immunostaining analysis for PFV-Gag. BHK-21 cells were grown on coverslips and infected with $1 \mathrm{ml}$ of cell-free culture supernatant for $6 \mathrm{~h}$. Then, the cells were incubated for the indicated length of time, fixed and permeabilized as indicated in the "Materials and Methods". After blocking, coverslips were successively incubated with anti-PFV-IN (1:40) and anti-Gag (1:100) for $1 \mathrm{~h}$. The coverslips were incubated for 30 min with a dilution of 1:80 (PFV-IN) and 1:150 (PFV-Gag) of FITCconjugated donkey anti-rabbit IgG secondary antibody and mounted with a drop of UltraCruz mounting medium onto a microslide. Fluorescence was detected by a fluorescence microscope.

in the cytoplasm and nucleus at 48 and $72 \mathrm{~h}$ post infection, as shown for the wild type. These results indicate that arginine at 
positions $305,313,315$, and 329 are directly involved in NLS function of PFV-IN, whereas amino acids at positions 308,318 , and 324 are not.

We examined Gag protein localization in the wild type, one defective mutant, and one non-defective mutant to further confirm PFV-Gag sub-cellular localization in BHK-21 cells. The culture conditions were the same and the infection was performed as described for PFV-IN localization. In good agreement with the data shown in the sub-cellular fractionation experiment (Fig. 3A), Gag fluorescence was exclusively observed in the nucleus at $24 \mathrm{~h}$ post infection in the wild type virus, and cytoplasmic and nuclear fluorescence increased at $48 \mathrm{~h}$ and 72 $\mathrm{h}$ post infection in the immunocytochemistry (Fig. 3C). As expected, the non-defective mutant showed a similar localization pattern as the Gag protein in wild type viruses. However we observed a deviant Gag localization behavior for the defective mutants and predominant nuclear fluorescence was observed at 24,48 , and $72 \mathrm{~h}$ post infection. No cytoplasmic fluorescence was observed. These localization patterns were similar to the Gag localization observed for sub-cellular fractionation of the defective mutants.

\section{DISCUSSION}

In this study, we described the identification of four critical basic amino acids as the NLS of the PFV-IN protein and their role in PFV replication and infection. By analyzing infected cells at 24 , 48 , and $72 \mathrm{~h}$ after viral challenge, we found that the NLS of virion-associated IN plays an important role in post-entry nuclear accumulation of newly synthesized IN but not Gag (Fig. 3). In addition, controlled studies demonstrated that disruption of the NLS did not inactivate many of the known activities and attributes of IN. Specifically, virion production and the incorporation of $\mathrm{IN}$, sub-cellular localization and processing of viral polyproteins, and reverse transcription were largely unaffected by changing the basic amino acids lysine or arginine to threonine or proline (Fig. 1).

The NLS of the PFV IN we found here was composed of four discontinuous, functional basic amino acids over a region of 26 amino acids. Although the NLS spans over a short peptide in the C-terminal region, it might play an essential role interacting with cellular co-factors that direct nuclear importation of incoming viral DNA. The interaction between HIV-1 IN and the lens epithelium-derived growth factor (LEDGF/p75) is well documented, and amino acids responsible for HIV-1 IN nuclear importation interact with LEDGF/p75 and target viral DNA of the host chromosome (Emiliani et al., 2005). Another study found a putative bipartite NLS of HIV-1 IN, comprising residues 186-188 and 211-219, that facilitates an interaction between IN and karyopherin $\alpha$ (Gallay et al., 1997). V165 and R166 within a 13residue peptide located in the core domain of HIV-1 IN, but not bipartite, are critical for nuclear importation (Bouyac-Bertoia et al., 2001). The nucleophilic determinant of FIV IN has been mapped to the highly conserved $\mathrm{HHCC}$ motif in the N-terminal domain to function as NLS that requires multimerization mediated by the HHCC motif, which, in turn, directly promotes the interaction between the FIV IN and the cellular proteins involved in nuclear importation (Woodward et al., 2003). Therefore, an analysis of the interactions between PFV IN and LEDGF/ p75, karyopherin $\alpha$ or such analogous cellular proteins could be an interesting target for future study.

The karyophilic determinant for avian sarcoma virus IN has been proposed to be a noncanonical NLS that maps to six basic amino acids located within residues $207-235$ of the pro- tein (Kukolj et al., 1997) and can be compared with the four basic amino acids residues that we mapped in the functional NLS of PFV-IN.

Lentiviruses have the ability to infect post-mitotic cells or cells arrested in the G/S phase, suggesting that the PIC traverses an intact nuclear membrane (Fassati, 2006; Katz et al., 2005; Suzuki and Craigie, 2007). The FV genome and Gag can enter the nuclei of growth-arrested cells, indicating the ability of the FV PIC to cross an intact nuclear membrane similar to that of lentiviruses (Lo et al., 2010). However, cells transduced with INdeficient vectors reveal no Gag or viral genome within the nuclei, suggesting that Gag alone is not critical for PIC transport to the nucleus, whereas IN is an absolute requirement (Lo et al., 2010). The study did not identify any dependency of Gag nuclear localization on IN in cycling cells. Another study suggested that Gag transport to the nucleus is totally independent of IN (Mullers et al., 2011), similar to our observations. We observed that viral Gag translocated to the nucleus even in the presence of all NLS inactivating mutants, whereas IN was predominantly present in the cytoplasm (Fig. 3). Therefore, we suggest that Gag translocation is independent of PFV-IN.

An NLS is present in both the Gag and IN proteins of FVs (Imrich et al., 2000; Schliephake and Rethwilm, 1994). The NLS in Gag serves as a nuclear transport signal resulting in higher accumulation in the nucleus. However, FV replication is independent of the Gag NLS (Yu et al., 1996); thus, supporting our findings.

The matrix (MA) domain of Gag and Vpr of lentiviruses has NLS sequences that are thought to be important for PIC importation into the nuclei of growth-arrested cells (Bukrinsky et al., 1993; Connor et al., 1995; Heinzinger et al., 1994). However, this notion remains controversial, as viruses with mutated MA and Vpr NLS sequences can infect nondividing cells; thus, refuting a major role for MA and Vpr in PIC translocation to the nucleus (Gallay, 1997; Kootstra and Schuitemaker, 1999).

We conclude that prototype FV IN contains multiple nuclear localization signals in its conserved C-terminal domain. Here, we described the identification of four critical basic amino acids as the NLS of the PFV-IN protein that were essential for PFV infection. An inactivating mutation on this NLS caused predominant IN cytoplasmic localization, which ultimately caused lack of integration into the viral genome and lack of replication. In contrast, localization of a consistent amount of Gag and IN for the defective mutants also indicates a lack of integration and lack of viral genome expression. The PFV is considered a permissive safe vector for gene therapy. Therefore, our results will be helpful for ongoing PFV studies.

\section{ACKNOWLEDGMENTS}

This study was supported by grant 2011-0011256 from the National Research Foundation of Korea funded by the Korean government. The authors deeply appreciate Drs. Maxine L. Linial and Eun-Gyung Lee (Fred Hutchinson Cancer Research Center, USA) for the generous gift of the FAB cell line and the protocol.

\section{REFERENCES}

Achong, B.G., Mansell, P.W., Epstein, M.A., and Clifford. P. (1971). An unusual virus in cultures from a human nasopharyngeal carcinoma. J. Natl. Cancer Inst. 46, 299-307.

Bouyac-Bertoia, M., Dvorin, J.D., Fouchier, R.A., Jenkins, Y., Meyer B.E., Wu, L.I., Emerman, M., and Malim, M.H. (2001). HIV-1 infection requires a functional integrase NLS. Mol. Cell 7, 10251035.

Brown, P.O. (1997). Integration. In Retroviruses, J.M. Coffin, S.H., 
Hughes, and H.E. Varmus, eds. (Cold Spring Harbor, N.Y.: Cold Spring Harbor Laboratory Press), pp. 161-204.

Bukrinsky, M.I., Haggerty, S., Dempsey, M.P., Sharova, N., Adzhubel, A., Spitz, L., Lewis, P., Goldfarb, D., Emerman, M., and Stevenson, M. (1993). A nuclear localization signal within HIV-1 matrix protein that governs infection of non-dividing cells. Nature 365, 666-669.

Bushman, F.D., Fujiwara, T., and Craigie, R. (1990). Retroviral DNA integration directed by HIV integration protein in vitro. Science 249, 1555-1558.

Connor, R.I., Chen, B.K., Choe, S., Landau, N.R. (1995). Vpr is required for efficient replication of human immunodeficiency virus type 1 in mononuclear phagocytes. Virology 206, 935-944.

Craigie, R. (2001). HIV integrase, a brief overview from chemistry to therapeutics. J. Biol. Chem. 276, 23213-23216.

Emiliani, S., Mousnier, A., Busschots, K., Maroun, M., Maele, B., Tempe, D., Vandekerckhove, L., Moisant, F., Ben-Slama, L., Witvrouw, M., et al. (2005). Integrase mutants defective for interaction with LEDGE/ p75 are impaired in chromosome tethering and HIV-1 replication. J. Biol. Chem. 280, 25517-25523.

Engelman, A. (1999). In vivo analysis of retroviral integrase structure and function. Adv .Virus Res. 52, 411-426.

Engelman, A., Mizuuchi, K., and Craigie, R. (1991). HIV-1 DNA integration: mechanism of viral DNA cleavage and DNA strand transfer. Cell 67, 1211-1221.

Enssle, J., Moebes, A., Heinkelein, M., Panhuysen, M., Mauer, B., Schweizer, M., Neumann-Haefelin, D., and Rethwilm, A. (1999). An active foamy virus integrase is required for virus replication. $\mathrm{J}$. Gen. Virol. 80, 1445-1452.

Epstein, M.A. (2004). Simian retroviral infections in human beings. Lancet 364, 138-139.

Fassati, A. (2006). HIV infection of non-dividing cells: a divisive problem. Retrovirology 3, 74 .

Fauquet, C.M., and Fargette, D. (2005). International Committee on Taxonomy of Viruses and the 3,142 unassigned species. Virol. J. 2, 64.

Gallay, P., Hope, T., Chin, D., and Trono, D. (1997). HIV-1 infection of nondividing cells through the recognition of integrase by the importin/karyopherin pathway. Proc. Natl. Acad. Sci. USA 94, 9825-9830.

Gorlich, D., and Kutay, U. (1999). Transport between the cell nucleus and the cytoplasm. Annu. Rev. Cell Dev. Biol. 15, 607-660.

Heinzinger, N.K., Bukrinsky, M.L., Haggerty, S.A., Ragland, A.M. Kewalramani, V., Lee, M.A., Gendelman, H.E., Ratner, M., Stevenson, M., and Emerman, M. (1994). The Vpr protein of human immunodeficiency virus type 1 influences nuclear localization of viral nucleic acids in nondividing host cells. Proc. Natl. Acad. Sci. USA 91, 7311-7315.

Herchenroder, O., Renne, R., Loncar, D., Cobb, E.K., Murthy, K.K. Schneider, J., Mergi, A., and Luciw, P.A. (1994). Isolation, cloning, and sequencing of simian foamy viruses from chimpanzees (SFVcpz): high homology to human foamy virus (HFV). Virology 201, 187-199.

Holden, P., and Horton, W.A. (2009). Crude subcellular fractionation of cultured mammalian cell lines. BMC Res. Notes 2, 243

Imrich, H., Heinkelein, M., Herchenröder, O., and Rethwilm, A. (2000). Primate foamy virus Pol proteins are imported into the nucleus. J. Gen. Virol. 81, 2941-2947.

Kang, S.Y., Ahn, D.G., Lee, C., Lee, Y.S., and Shin, C.-G. (2008) Functional nucleotides of U5 LTR determining substrate specificity of prototype foamy virus integrase. J. Microbial. Biotechnol. 18, 1044-1049.

Katz, R.A., Greger, J.G., and Skalka, A.M. (2002). Transduction of interphase cells by avian sarcoma virus. J. Virol. 76,5422-5434

Katz, R.A., Greger, J.G., and Skalka, A.M. (2005). Effects of cell cycle status on early events in retroviral replication. J. Cell. Biochem. 94, 880-889.

Kootstra, N.A., and Schuitemaker, H. (1999). Phenotype of HIV-1 lacking a functional nuclear localization signal in matrix protein of Gag and Vpr is comparable to wild-type HIV-1 in primary macrophages. Virology 253, 170-180.

Kukolj, G., Jones, K.S., and Skalka, A.M. (1997). Subcellular localization of avian sarcoma virus and human immunodeficiency virus type 1 integrases. J. Virol. 71, 843-847.

Lecellier, C.H, and Saib, A. (2000). Foamy viruses: between retroviruses and pararetroviruses. Virology 271, 1-8.
Lee, H.S., Kang, S.Y., and Shin, C.-G. (2005). Characterization of the functional domains of human foamy virus integrase using chimeric integrases. Mol. Cells 19, 246-255.

Linial, M.L. (1999). Foamy viruses are unconventional retroviruses. J. Virol. $73,1747-1755$.

Linial, M.L. (2007). Foamy viruses. In Field Virology, D.M. Knipe and P.M. Howley, eds. (Philadelphia, PA: Lippincott Williams \& Wilkins), pp. 2245-2262.

Lo, Y.T., Tian, T., Nadeau, P.E., Park, J., and Mergia, A. (2010) The foamy virus genome remains unintegrated in the nuclei of G1/S phase-arrested cells, and integrase is critical for preintegration complex transport into the nucleus. J. Virol. 84, 28322842.

Löchelt, M., and Flugel, R.M. (1996). The human foamy virus pol gene is expressed as a Pro-Pol polyprotein and not as a GagPol fusion protein. J. Virol. 70, 1033-1040.

Löchelt, M., Yu, S.F., Linial, M.L., and Flügel, R.M. (1995). The human foamy virus internal promoter is required for efficient gene expression and infectivity. Virology 206, 601-610.

Mahnke, C., Löchelt, M., Bannert, B., and Flügel, R.M. (1990). Specific enzyme-linked immunosorbent assay for the detection of antibodies to the human spumavirus. J. Virol. Methods 29,1322.

Mattaj, I.W., and Englmeier, L. (1998). Nucleocytoplasmic transport: the soluble phase. Annu. Rev. Biochem. 67, 265-306.

Mergia, A., and Heinkelein, M. (2003). Foamy virus vectors. Curr. Top. Microbiol. Immunol. 277, 131-159.

Miller, M.D., Farnet, C.M., and Bushman, F.D. (1997). Human immunodeficiency virus type 1 preintegration complexes: studies of organization and composition. J. Virol. 71, 5382-5390.

Mullers, E., Stirnnagel, K., Kaulfuss, S., and Lindemann, D. (2011). Prototype foamy virus gag nuclear localization: a novel pathway among retroviruses. J. Virol. 85, 9276-9285.

Patton, G.S., Erlwein, O., and McClure, M.O. 2004. Cell-cycle dependence of foamy virus vectors. J. Gen. Virol. 85, 2925-2930.

Pearl, L.H., and Taylor, W.R. (1987). A structural model for the retroviral proteases. Nature 329, 351-354.

Petit, C., Schwartz, O., and Mammano, F. (2000). The karyophilic properties of human immunodeficiency virus type 1 integrase are not required for nuclear import of proviral DNA. J. Virol. 74 7119-7126.

Rethwilm, A. (1996). Unexpected replication pathways of foamy viruses. J. Acquir. Immune. Defic. Syndr. Hum. Retrovirol. 13 Supp $1, \mathrm{~S} 248-253$

Rethwilm, A. (2005). Foamy viruses. In Virology, V.T. Meulen and B.W.J. Mahy, eds. (London, United Kingdom: Topley \& Wilson), pp. 1304-1321

Rinke, C.S., Boyer, P.L., Sullivan, M.D., Hughes, S.H., and Linial, M.L. (2002). Mutation of the catalytic domain of the foamy virus reverse transcriptase leads to loss of processivity and infectivity. J. Virol. 76, 7560-7570.

Rothnie, H.M., Chapdelaine, Y., Chapdelaine, Y., and Hohn, T. (1994). Pararetroviruses and retroviruses: a comparative review of viral structure and gene expression strategies. Adv. Virus. Res. 44, 1-67.

Schliephake, A.W., and Rethwilm, A. (1994). Nuclear localization of foamy virus Gag precursor protein. J. Virol. 68, 4946-4954.

Schweizer, M., Turek, R., Hahn, H., Schliephake, A., Netzer, K.O., Eder, G., Reinhardt, M., Rethwilm, A., and Neumann-Haefelin, D. (1995). Markers of foamy virus infections in monkeys, apes, and accidentally infected humans: appropriate testing fails to confirm suspected foamy virus prevalence in humans. AIDS Res. Hum. Retroviruses 11, 161-170.

Suzuki, Y., and Craigie, R. (2007). The road to chromatin - nuclear entry of retroviruses. Nat. Rev. Microbiol. 5, 187-196.

Tobaly-Tapiero, J., Bittoun, P., Lehmann-Che, J., Delelis, O., Giron, M.L., de Thé, H., and Saïb, A. (2008). Chromatin tethering of incoming foamy virus by the structural Gag protein. Traffic 9 , 1717-1727.

Wei, S.Q., Mizuuchi, K., and Craigie, R. (1997). A large nucleoprotein assembly at the ends of the viral DNA mediates retroviral DNA integration. EMBO J. 16, 7511-7520.

Woodward, C.L., Wang, C., Dixon, W.J., Htun, H., and Chow, S.A. (2003). Subcellular localization of feline immunodeficiency virus integrase and mapping of its karyophilic determinant. J. Virol. 77 4516-4527. 
PFV Integrase NLS for Infection

Md. Alamgir Hossain et al.

Yu, S.F., and Linial, M.L. (1993). Analysis of the role of the bel and bet open reading frames of human foamy virus by using a new quantitative assay. J. Virol. 67, 6618-6624

Yu, S.F., Edelmann, K., Strong, R.K., Moebes, A., Rethwilm, A., and Linial, M.L. (1996). The carboxyl terminus of the human foamy virus Gag protein contains separable nucleic acid binding and nuclear transport domains. J. Virol. 70, 8255-8262.

Yu, S.F., Sullivan, M.D., and Linial, M.L. (1999). Evidence that the human foamy virus genome is DNA. J. Virol. $73,1565-1572$. 